\title{
Evaluation of Very High- and Very Low-Dose Intravitreal Aflibercept in Patients with Neovascular Age-Related Macular Degeneration
}

\author{
Quan Dong Nguyen,, Peter A. Campochiaro,, Syed Mahmood Shah,, David J. Browning, ${ }^{2}$ Henry L. Hudson, ${ }^{3}$ \\ Peter L. Sonkin, ${ }^{4}$ Seenu M. Hariprasad, ${ }^{5}$ Peter K. Kaiser, ${ }^{6}$ Jason Slakter, ${ }^{7}$ Julia A. Haller, ${ }^{1}$ \\ Diana V. Do, ${ }^{1}$ William Mieler, ${ }^{5}$ Karen Chu, ${ }^{8}$ Avner Ingerman, ${ }^{8}$ Robert Vitti, ${ }^{8}$ \\ Alyson J. Berliner, ${ }^{8}$ Jesse Cedarbaum, ${ }^{8}$ and the CLEAR-IT 1 Investigators
}

\begin{abstract}
Purpose: To determine bioactivity and duration of effect of intravitreal aflibercept injection (also known as vascular endothelial growth factor Trap-Eye) for neovascular age-related macular degeneration (AMD).

Methods: In this double-masked, phase 1 study, 28 patients with lesions $\leq 12$ disc areas, $\geq 50 \%$ active choroidal neovascularization $(\mathrm{CNV})$, and best corrected visual acuity (BCVA) $\leq 20 / 40$ were randomized 1:1 to a single intravitreal injection of aflibercept 0.15 or $4 \mathrm{mg}$. The primary end point was the change from baseline in central retinal/lesion thickness (CR/LT) at week-8. Secondary outcomes were the change from baseline BCVA, the change in CNV lesion size and area of leakage, and proportion of patients requiring repeat injection at 8 weeks. Results: Mean percent decrease in CR/LT for the 4-mg and 0.15-mg groups was, respectively, 34.2 versus 13.3 at week $4(P=0.0065), 23.8$ versus 5.9 at week $6(P=0.0380)$, and $25.2 \%$ versus $11.3 \%$ at week $8(P=0.150)$. The $4-\mathrm{mg}$ group gained a mean of 4.5 letters in BCVA (6/14 patients gaining $\geq 10$ letters) versus 1.1 letters in $0.15-\mathrm{mg}$ group ( $1 / 14$ gaining $\geq 10$ letters) at week 8 . Fewer patients needed retreatment in the 4 -mg group at week 8 . No serious adverse event or ocular inflammation was reported in either group.

Conclusions: Intravitreal aflibercept $4 \mathrm{mg}$ had a safety profile similar to that of the very low dose $0.15 \mathrm{mg}$, and was well-tolerated. The 4-mg dose significantly reduced foveal thickening at weeks 4 and 6, significantly improved BCVA at weeks 6 , and reduced the need for repeat injection after 8 weeks compared with intravitreal aflibercept $0.15 \mathrm{mg}$ in neovascular AMD patients.
\end{abstract}

\section{Introduction}

V ASCULAR ENDOTHELIAL GROWTH FACTOR (VEGF) is a critical stimulus for subretinal and choroidal neovascularization $(\mathrm{CNV})$ in animal models ${ }^{1-4}$ and its importance in neovascular age-related macular degeneration (AMD) has been confirmed. 5,6 Ranibizumab, a humanized monoclonal antibody that binds all isoforms of VEGF-A has been shown in numerous studies to stabilize and improve best corrected visual acuity (BCVA) in patients with neovascular $\mathrm{AMD}, 5,6$ but frequent intraocular injections are generally required to maintain those benefits. Development of VEGF antagonists that are more potent and/or have a longer duration of action is a high priority.

Aflibercept is a recombinant protein in which the binding domains of VEGF receptors 1 and 2 have been combined with the Fc portion of IgG. The receptor portion of the molecule has a very high affinity for all VEGF-A isoforms $\left(\mathrm{K}_{\mathrm{d}}<1 \mathrm{pM}\right)$, Placental Growth Factor (PlGF)-1 and -2 , and VEGF-B. ${ }^{7}$ Therefore, aflibercept is distinguished from ranibizumab by its higher binding affinity for all VEGF-A isoforms and its ability to inhibit other VEGF family members. A

\footnotetext{
${ }^{1}$ The Wilmer Eye Institute, The Johns Hopkins University School of Medicine, Baltimore, Maryland.

${ }^{2}$ Charlotte Eye, Ear, Nose, and Throat Associates PA, Charlotte, North Carolina.

${ }^{3}$ Retina Centers PC, Tucson, Arizona.

${ }^{4}$ Tennessee Retina PC, Nashville, Tennessee.

${ }^{5}$ University of Chicago, Chicago, Illinois.

${ }^{6}$ Cleveland Clinic Foundation, Cleveland, Ohio.

${ }^{7}$ Vitreous Retina Macula Consultants of New York, New York, New York.

${ }^{8}$ Regeneron Pharmaceuticals, Inc., Tarrytown, New York.
} 
randomized, multicenter, placebo-controlled clinical trial investigating the effect of intravenous aflibercept in patients with neovascular AMD showed elimination of about $60 \%$ of excess retinal thickness after either single or multiple infusions. ${ }^{8}$ The maximum tolerated dose of intravenous aflibercept in this study population was $1.0 \mathrm{mg} / \mathrm{kg}$; at $3 \mathrm{mg} / \mathrm{kg}$, hypertension, and proteinuria, which are class effects of systemic anti-VEGF therapy, were noted. Thus, alternative routes of delivery were investigated to increase the duration of therapeutic effect and minimize the potential for adverse events (AEs).

Intravitreal aflibercept injection (also known as VEGF Trap-Eye) is a formulation utilizing ultrapurified aflibercept in an iso-osmotic combination of buffers compatible with intraocular administration. Preclinical and model studies have suggested that intravitreal aflibercept may maintain a significant intravitreal VEGF-binding activity for several weeks after a single injection at doses of $0.5 \mathrm{mg}$ and $4 \mathrm{mg}$ in rabbits and humans, respectively (Furfine, et al. Association for Research in Vision \& Ophthalmology. Invest Ophthalmol Vis Sci, 2006; 47:E-Abstract 1430). ${ }^{9}$ In a dose-ranging phase 1 trial of 21 patients with advanced neovascular AMD, a single intravitreal injection of aflibercept at doses from $0.05 \mathrm{mg}$ to $4 \mathrm{mg}$ was well-tolerated and showed evidence of bioactivity. ${ }^{10}$ Three of the 6 patients in this study treated with one intravitreal dose of 2 or $4 \mathrm{mg}$ showed improvement in BCVA $\geq 3$ lines at the 6-week primary end point. ${ }^{10}$ The current study is a prospective randomized trial comparing the visual and anatomic effects of intravitreal injection of $4 \mathrm{mg}$ or $0.15 \mathrm{mg}$ of aflibercept in eyes with neovascular AMD, designed to evaluate the bioactivity, safety and tolerability, and duration of action of the 2 doses of aflibercept.

\section{Methods}

The study was a prospective, double-masked, randomized clinical trial comparing a single intravitreal injection of 0.15 or $4 \mathrm{mg}$ of aflibercept in patients with neovascular AMD. The study was conducted at 5 sites and in compliance with the Declaration of Helsinki, US Code 21 of Federal Regulations, and the Harmonized Tripartite Guidelines for Good Clinical Practice (1996), and was reviewed and approved by all appropriate Institutional Review Boards. All participating patients provided written informed consent.

\section{Study population}

Participants were men or women of any ethnicity, aged 50 years or older, with the diagnosis of neovascular AMD with subfoveal $\mathrm{CNV} \leq 12$ disc areas in one or both eyes (measured according to the protocol of the Macular Photocoagulation Study ${ }^{11}$ ) showing leakage on fluorescein angiography (FA), BCVA of $20 / 40$ to $20 / 320$, and central subfield thickness $\geq 250 \mu \mathrm{m}$ measured by optical coherence tomography (OCT), with no prior history of intravitreal injection of aflibercept or bevacizumab or ranibizumab. Prior treatment with the following therapies in the study eye was only excluded if treatment fell within the noted time: laser photocoagulation or photodynamic therapy or intravitreal triamcinolone within 12 weeks, intravitreal injection of pegaptanib within 8 weeks, and periocular corticosteroids in the study eye within 6 months of enrollment (See Supplementary Table S1; Supplementary Data are available online at www.liebertpub .com/jop, for a complete eligibility criteria listing).

\section{Schedule of visits, interventions, and assessments}

Patients were evaluated at screening, day 1 (baseline), day 3 , and weeks 1, 2, 4, 6, 8, and 12. Patients were randomly assigned to receive the intravitreal injection of either $0.15 \mathrm{mg}$ or $4 \mathrm{mg}$ aflibercept in a 1:1 ratio. The initial intravitreal injection of $0.15 \mathrm{mg}$ or $4 \mathrm{mg}$ aflibercept was given at the day 1 baseline visit; the technique for injection is described below. At week 8, patients had the option of remaining in the masked phase through day 85 or entering a 1-year open-label extension phase, during which patients were evaluated for retreatment based on the following criteria: (1) an increase in central retinal thickness of $\geq 100 \mu \mathrm{m}$ as measured by OCT, (2) a loss of $\geq 5$ Early Treatment Diabetic Retinopathy Study (ETDRS) letters in conjunction with recurrent fluid as indicated by OCT, (3) persistent fluid as indicated by OCT, (4) new onset classic neovascularization, (5) new or persistent leakage on FA, or (6) new macular hemorrhage. Safety and efficacy for the masked phase of this study were evaluated at week 8 and are reported herein.

\section{Intravitreal administration of aflibercept}

Intravitreal injections of aflibercept were performed according to a prespecified protocol. A sterile lid speculum was inserted, topical anesthesia was applied, and the conjunctiva was irrigated with $5 \%$ povidone iodine. After additional local anesthesia, a 30-gauge needle was inserted through the pars plana, and the assigned dose of aflibercept was injected into the vitreous cavity. Funduscopic examination was then performed to confirm retinal perfusion, and patients were observed for $1 \mathrm{~h}$ or until intraocular pressure was $\leq 21 \mathrm{~mm} \mathrm{Hg}$.

\section{Study assessments}

Patients were closely monitored for safety and tolerability using the following assessments and procedures: BCVA, slitlamp biomicroscopy, indirect ophthalmoscopy, tonometry, AE reporting, vital signs, physical examinations, serum electrolytes, creatinine, quantitative protein determination in 24-h urine specimens, and measurement of serum neutralizing antibodies directed against aflibercept. Stereoscopic color fundus photographs and FA were performed at baseline and week 6 and OCT was performed at each study visit. OCT was performed using StratusOCT ${ }^{\circledR}$ (Carl Zeiss Meditec, Dublin, CA) to obtain 6-mm fast macular thickness maps and $6 \times 6-\mathrm{mm}$ crosshair scans. All images were evaluated by masked graders at the Digital OCT Reading Center (DOCTR, Cleveland, $\mathrm{OH})$. Foveal thickness, defined as the mean height of the neurosensory retina in a central 1-mm-diameter area, and total macular volume were automatically computed by the StratusOCT ${ }^{\circledR}$ software version 4 . Central retinal/lesion thickness (CR/LT) was manually computed by masked graders. CR/LT was defined as the distance between the inner limiting membrane and the inner border of the retinal pigment epithelium/choriocapillaris complex, including any subretinal fluid and thickness of any observable choroidal neovascular membrane or scar tissue in the central $1 \mathrm{~mm}$ of the posterior pole scan. A posterior pole scan was obtained, consisting of a high-resolution 7-mm scan from a single scan line from the meridian of the optic disc margin, declined at a 5-degree angle through the presumed foveal center. The placement of the scan line was based on anatomic landmarks as visualized by a trained, certified operator to offer better registration. FA was 
Table 1. Demographics and Baseline Characteristics of the Study Population

\begin{tabular}{|c|c|c|c|c|c|c|}
\hline & \multicolumn{3}{|c|}{0.15 mg VEGF Trap $(\mathrm{n}=14)$} & \multicolumn{3}{|c|}{$4.0 m g$ VEGF Trap $(\mathrm{n}=14)$} \\
\hline & $M e a n \pm S D$ & Median & Range & Mean $\pm S D$ & Median & Range \\
\hline Age & $75.6 \pm 7.76$ & 76 & $55-87$ & $76.9 \pm 6.85$ & 78.5 & $66-89$ \\
\hline Gender (M:F) & & $8: 6$ & & & $7: 7$ & \\
\hline Study Eye (OD:OS) & & $6: 8$ & & & $6: 8$ & \\
\hline $\mathrm{BCVA}^{\mathrm{a}}$ & $49.7 \pm 15.48$ & 46.5 & $27-75$ & $51.8 \pm 13.61$ & 54.5 & $22-73$ \\
\hline Snellen Equivalent & $20 / 100$ & $20 / 125+1$ & $20 / 320+2-20 / 32$ & $20 / 100+1$ & $20 / 80-1$ & $20 / 400+2-20 / 32-2$ \\
\hline $\begin{array}{c}\text { Central Retinal/Lesion } \\
\text { Thickness (CR/LT) }\end{array}$ & $493.9 \pm 170.69$ & 475.8 & $212-862.5$ & $506.0 \pm 156.02$ & 474 & $282-873$ \\
\hline $\begin{array}{l}\text { \# of Prior Treatments } \\
\text { in Study Eye (PDT } \pm \\
\text { steroids, pegaptanib, } \\
\text { bevacizumab) }\end{array}$ & \multicolumn{3}{|c|}{ None $=6 ;>1=8$} & \multicolumn{3}{|c|}{ None $=9 ;>1=5$} \\
\hline \multicolumn{7}{|l|}{ Lesion Type } \\
\hline $\begin{array}{l}\text { Classic/ } \\
\text { Predominantly } \\
\text { Classic }\end{array}$ & \multicolumn{3}{|c|}{1} & \multicolumn{3}{|c|}{5} \\
\hline Minimally Classic & \multirow{2}{*}{\multicolumn{3}{|c|}{$\begin{array}{c}2 \\
11\end{array}$}} & \multicolumn{3}{|c|}{3} \\
\hline Occult & & & & \multicolumn{3}{|c|}{6} \\
\hline
\end{tabular}

${ }^{a}$ ETDRS letters read as measured by EVA (electronic visual acuity).

BCVA, best corrected visual acuity; PDT, photodynamic therapy; CNV, choroidal neovascularization; FA, fluorescein angiography.

performed in customary fashion. The Digital Angiographic Reading Center (DARC, New York, NY) provided a modified image acquisition protocol and monitored compliance by site visits. Digital angiogram images were evaluated by masked graders at DARC.

\section{Data analysis}

The primary objective of this phase 1 study was to compare the safety, tolerability, and bioactivity of $0.15 \mathrm{mg}$ versus $4 \mathrm{mg}$ intravitreal injections of aflibercept. The primary efficacy outcome was the change from baseline to week 8 in CR/LT. The primary analysis consisted of a paired $t$-test of the significance of change from baseline to week 8 by pooling observations from the 2-dose groups combined. Conditional on statistical significance of the 1-sample analysis of the pooled group, an analysis of covariance (ANCOVA) was conducted to compare the 2-dose groups. The ANCOVA model was a main-effects model, including baseline CR/LT as a covariate and treatment as a factor. Secondary outcomes were the change from baseline to week 8 in BCVA, the change from baseline to week 6 in CNV lesion size and area of leakage, and the proportion of patients requiring repeat injection at 8 weeks. The between-group difference in proportion of patients gaining $\geq 10$ letters in BCVA was analyzed using the Fisher's exact test. Missing values were imputed using the last observation carried forward method.

\section{Results}

\section{Patient disposition and demographics}

Overall, 28 patients were enrolled and 14 each were randomized to receive either $0.15 \mathrm{mg}$ or $4 \mathrm{mg}$ intravitreal aflibercept; all 28 patients were treated as randomized. The baseline characteristics of the 28 patients included in the study are listed in Table 1. Fifteen were male, 27 were
Caucasian, and the mean age for all patients combined was 76.3 years. Although the majority of the patients had received prior treatment(s) for neovascular AMD, an effort was made to determine the presence of classic and/or occult CNV within lesions; history of prior treatments and lesion types by group are also given in Table 1 .

\section{Safety}

There were no ocular serious adverse events (SAEs) and no significant intraocular inflammation in any study eye. Common ocular AEs are given in Table 2. Conjunctival hemorrhage, change in refractive state, and reduced visual acuity were the most common AEs, and rates were similar between the 2-dose groups.

\section{$C R / L T$ on $O C T$}

Due to the presence of significant artifacts when using the automated fast macular thickness OCT scans, which rendered many scans unreadable, a manual measurement was utilized: (CR/LT), the combined thickness of CNV, subretinal fluid, and overlying retina in the center of the macula. Measurements were performed by masked graders (as described in Materials, Subjects, and Methods section). The mean change from baseline in CR/LT for the pooled groups at weeks 4,6 and 8 was $-128.5 \mu \mathrm{m}(P<0.0001)$, $-79.7 \mu \mathrm{m} \quad(P=0.0018)$, and $-97.1 \mu \mathrm{m} \quad(P=0.0006)$, respectively. This decrease represented a mean $18.3 \%$ reduction in CR/LT 8 weeks following a single intravitreal injection of aflibercept.

The mean absolute and percentage decrease in CR/LT of the study eye from baseline was consistently greater in the 4 -mg group than in the 0.15 -mg group. The mean percentage decrease in CR/LT for the 4-mg and 0.15-mg groups was, respectively, $34.2 \%$ versus $13.3 \%$ at week $4,23.8 \%$ 
Table 2. Adverse Events Occurring at a Frequency of > 5\% of the Overall Group [n, $(\%)$ ]

\begin{tabular}{lcrr}
\hline & All $(\mathrm{n}=28)$ & 0.15-mg Group (n=14) & $4-m g$ Group (n=14) \\
\hline Conjunctival hemorrhage & $20(71.4)$ & $10(71.4)$ & $10(71.4)$ \\
Refraction disorder & $9(32.1)$ & $4(28.6)$ & $5(35.7)$ \\
Reduced visual acuity & $4(14.3)$ & $1(7.1)$ & $3(21.4)$ \\
Anterior chamber cell & $2(7.1)$ & $1(7.1)$ & $1(7.1)$ \\
Cataract & $2(7.1)$ & $1(7.1)$ & $1(7.1)$ \\
Increased intraocular pressure & $2(7.1)$ & $1(7.1)$ & $1(7.1)$ \\
Ocular hyperemia & $2(7.1)$ & $1(7.1)$ & $1(7.1)$ \\
Vitritis & $2(7.1)$ & $1(7.1)$ & $1(7.1)$ \\
\hline
\end{tabular}

versus $5.9 \%$ at week 6 , and $25.2 \%$ versus $11.3 \%$ at week 8 (Fig. 1A). The percent difference between groups was statistically significant (by ANCOVA) at week $4(P=0.0065)$ and week $6(P=0.0380)$, but not at week $8 \quad(P=0.150)$ (Fig. 1A).

\section{Best corrected visual acuity}

The mean change from baseline in BCVA for the pooled groups at weeks 4,6 , and 8 was 3.6 letters $(P=0.0438), 4.8$ letters $(P=0.0237)$, and 2.8 letters $(P=0.1632)$, respectively.
FIG. 1. (A) Mean percentage change from baseline in combined lesion and foveal thickness between the 0.15 -mg and 4-mg doses. The p-values were calculated for the difference between the 2 doses and designated as $\left(^{*}\right)$ at week $6(P=0.038)$ and $(\dagger)$ at week $8(P=0.150)$. (B) Mean change from baseline in ETDRS visual acuity score between the $0.15-\mathrm{mg}$ and 4-mg doses. The $\mathrm{p}$-values were calculated for the difference between the 2 doses and designated as $\left(^{*}\right)$ at week $6(P=0.030)$ and $(\dagger)$ at week $8 \quad(P=0.383)$. ETDRS $=$ Early Treatment Diabetic Retinopathy Study. The arrow $(\downarrow)$ indicates opportunity for retreatment with vascular endothelial growth factor (VEGF) Trap-Eye at week 8 for patients in both the 0.15 -mg and 4-mg groups.

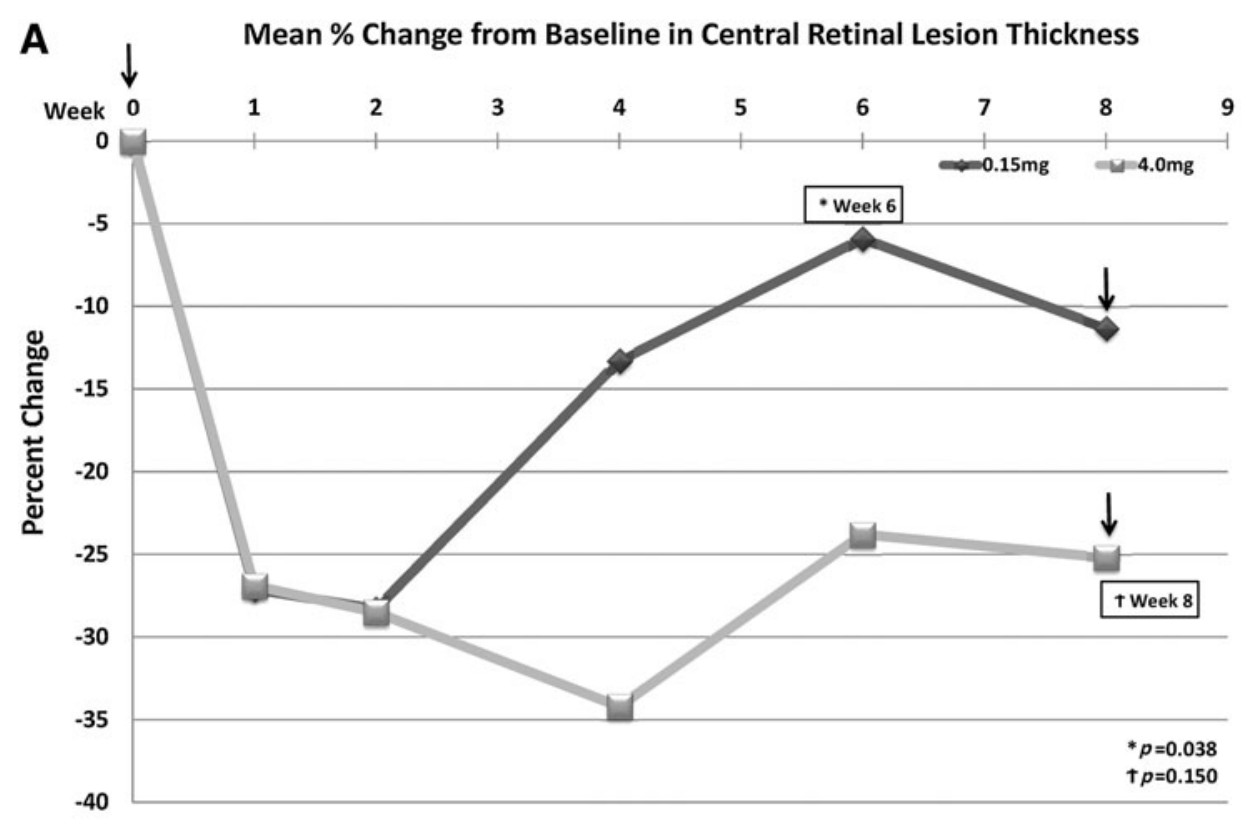

B

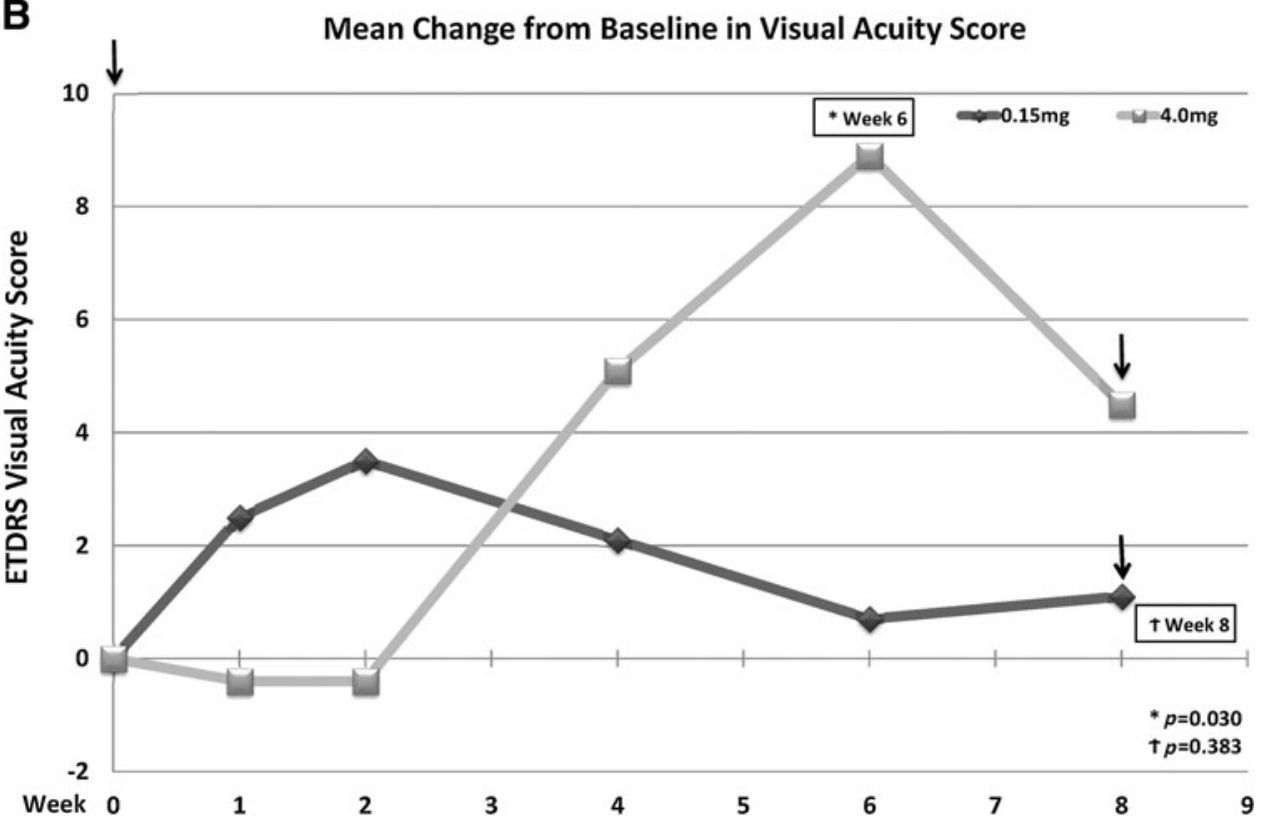




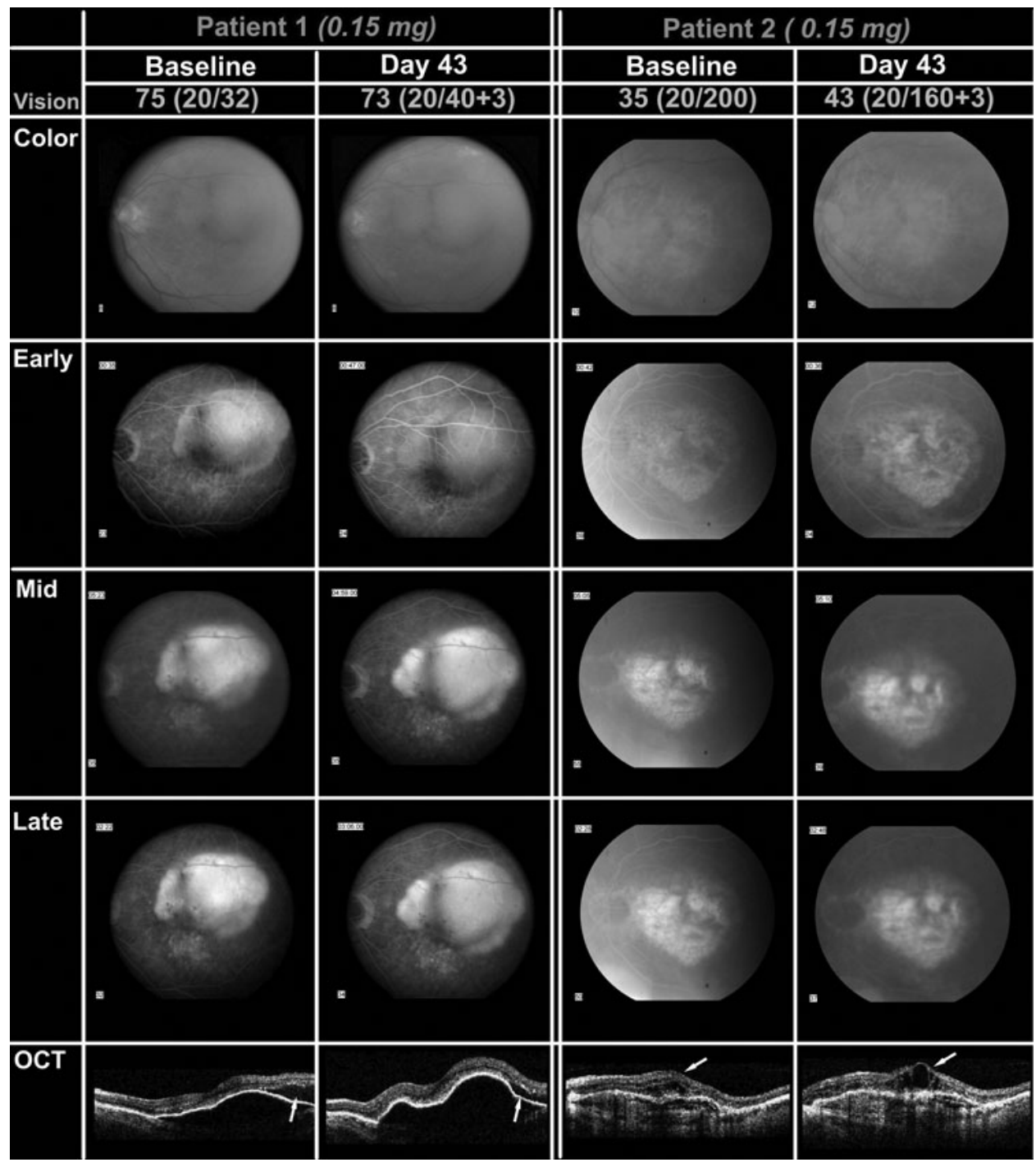

FIG. 2. Color photography, fluorescein angiography (FA), and optical coherence tomography (OCT) for 2 randomly selected patients who were randomized to receive the 0.15-mg dose of VEGF TrapEye: patients 1 and 2 . In patient 1, there was occult choroidal neovascularization (CNV) with fibrovascular pigment epithelial detachment seen on color photographs and $\mathrm{FA}$, and OCT revealed corresponding retinal pigment epithelial (RPE) detachment with subretinal fluid. At week 6 , there was persistent CNV leakage and elevation of the RPE on OCT. However, there was a small decrease in the amount of subretinal fluid seen on OCT. In patient 2, color fundus photography and FA revealed leakage from subfoveal CNV with cystoid retinal edema on OCT at the baseline visit. At week 6, there was persistent $\mathrm{CNV}$ leakage and an increase in the cystoid retinal edema seen on OCT.
At week 8,20 of 28 patients $(71 \%)$ had a gain in visual acuity of 0 or more letters, and 8 patients $(29 \%)$ had a loss. Six of the 8 patients $(75 \%)$ who had a decrease in visual acuity were in the 0.15 -mg group.

Beginning at week 4, visual acuity in the 4-mg group was consistently better than in the $0.15-\mathrm{mg}$ group. Nine of 14 patients $(64 \%)$ in the 0.15 -mg group and 11 of 14 patients $(79 \%)$ in the 4-mg group showed stable or improved BCVA at 8 weeks. The mean improvement in BCVA between baseline and week 6 was 0.7 letters in the $0.15-\mathrm{mg}$ group and 8.9 letters in the $4-\mathrm{mg}$ group $(P=0.0306)$. Six of 14 patients $(43 \%)$ in the 4-mg dose group had a gain of 10 or more letters compared to only 1 of $14(7 \%)$ in the $0.15-\mathrm{mg}$ group $(P=0.0768)$. At week 8 , the difference between the groups was less pronounced, although the 4-mg group had a better visual acuity gain (4.5 letters in the 4-mg group and 1.1 letters in the $0.15-\mathrm{mg}$ group, $P=0.3831$ ) (Fig. 1B).

\section{Retreatment at week 8}

Based on the criteria defined in the Materials, Subjects, and Methods section, patients were evaluated by the Investigator at week 8 for a possible second intravitreal injection of aflibercept. Among the 14 patients injected with $4 \mathrm{mg}$ of intravitreal aflibercept at baseline, 4 patients (29\%) were redosed at week 8 . On the other hand, among the 14 patients treated with $0.15 \mathrm{mg}$ of intravitreal aflibercept at baseline, 10 patients $(71 \%)$ were redosed at this visit $(P=0.0570$; Fig. 1$)$. Persistent fluid was the most common reason for retreatment in both the 4-mg and 0.15-mg groups.

\section{Representative Selected Cases}

Stereoscopic color fundus photography and FA were performed at baseline and week 6 , and OCT was performed at each study visit for all 28 patients in the study. FA and OCT images were then sent to DARC and DOCTR for formal analyses, respectively. Two patients were selected randomly from each of the 2-dose groups and are discussed in details to illustrate the bioactivity of intravitreal aflibercept.

Patients 1 and 2 were selected from the 0.15-mg group. Patient 1 was a 72-year-old man who had CNV in the left eye, which was treated previously with pegaptanib. Baseline BCVA was 20/32. Occult CNV with a fibrovascular pigment epithelial detachment was seen on color photographs and FA. OCT revealed a corresponding retinal pigment epithelial (RPE) detachment with subretinal fluid. At week 6 BCVA was 20/40, and there was persistent CNV leakage and elevation of the RPE on OCT. However, there was a small decrease in the amount of subretinal fluid seen on OCT (Fig. 2). 
FIG. 3. Color photography, FA, and OCT for 2 randomly selected patients who were randomized to receive the 4-mg dose of VEGF Trap-Eye: patients 3 and 4 . In patient 3 , color fundus photography and FA revealed leakage from subfoveal occult CNV with cystoid retinal edema and a lamellar hole on OCT at the baseline visit. At week 6 , there was no CNV leakage on FA, and resolution of the cystoid retinal edema on OCT. In patient 4 , there was predominantly classic $\mathrm{CNV}$ seen on color photograph and FA at baseline. The corresponding OCT showed subretinal fluid and intraretinal edema. At week 6, there was a marked reduction in $\mathrm{CNV}$ leakage and a significant decrease in subretinal fluid and intraretinal edema.
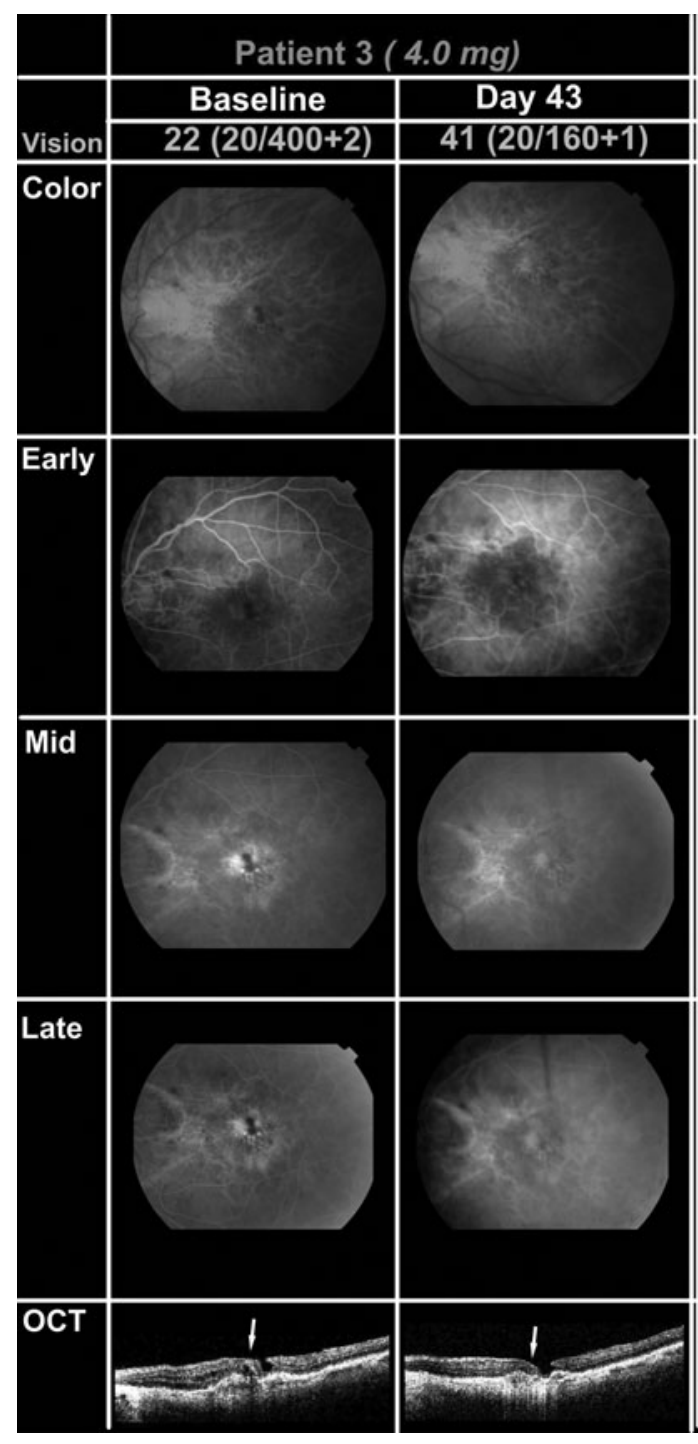

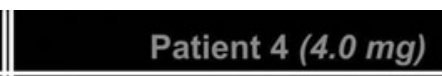

\begin{tabular}{|c|}
\hline Baseline \\
\hline $58(20 / 80+3)$ \\
\hline
\end{tabular}

\begin{tabular}{|c|}
\hline Day 43 \\
\hline $52(20 / 100+2)$ \\
\hline
\end{tabular}
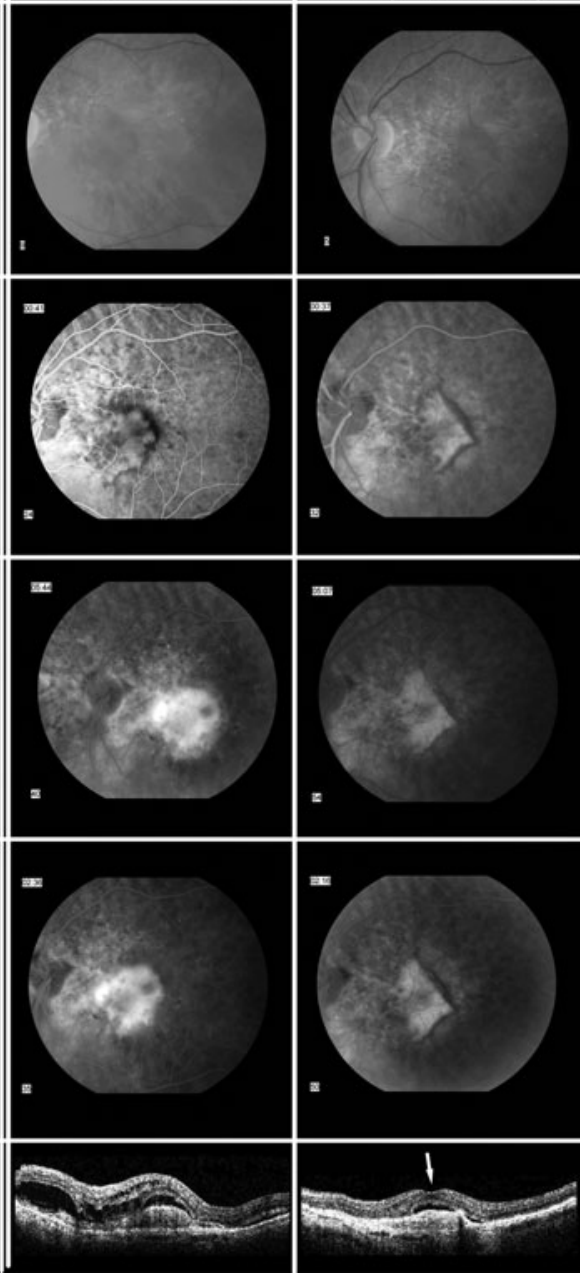

Patient 2 was a 78-year-old man who had CNV in the left eye, which was treated previously with both ranibizumab and pegaptanib. Baseline BCVA was 20/200. At the baseline visit, color fundus photography and FA revealed leakage from subfoveal $\mathrm{CNV}$, with cystoid retinal edema apparent on OCT. At week 6, BCVA was 20/160. At this visit, there was persistent $\mathrm{CNV}$ leakage and an increase in the cystoid retinal edema seen on OCT (Fig. 2).

Patients 3 and 4 were selected from the 4-mg group of intravitreal aflibercept. Patient 3 was an 81-year-old man who had $\mathrm{CNV}$ in the left eye, which was treated previously with verteporfin photodynamic therapy. Baseline BCVA was $20 / 400$. At the baseline visit, color fundus photography and FA revealed leakage from subfoveal occult $\mathrm{CNV}$, with cystoid retinal edema and a lamellar hole apparent on OCT. At week 6, BCVA was 20/160. At this visit, there was no CNV leakage with complete resolution of the cystoid retinal edema seen on OCT (Fig. 3).

Patient 4 was an 86-year-old woman who had CNV in the left eye, which was treatment naive. Baseline BCVA was 20/ 80. There was predominantly classic CNV seen on color photograph and FA at baseline. The corresponding OCT showed subretinal fluid and intraretinal edema. At week 6,
BCVA was 20/100. At this visit, there was a marked reduction in CNV leakage and a significant decrease in subretinal fluid and intraretinal edema (Fig. 3).

\section{Discussion}

A previous phase 1 study has demonstrated that an intravitreal injection of up to $4 \mathrm{mg}$ aflibercept in patients with AMD was well tolerated with no ocular inflammation. ${ }^{10}$ The current phase 1 study has confirmed that eyes treated with $4 \mathrm{mg}$ intravitreal aflibercept showed no evidence of inflammation and were indistinguishable from eyes injected with $0.15 \mathrm{mg}$ of intravitreal aflibercept with regard to safety and tolerability. The safety and tolerability profiles of both doses were identical during the 8-week study, with AEs generally being mild-to-moderate and self-limited. The most common $\mathrm{AE}$ was conjunctival hemorrhage, which is common to all drugs administered by an intravitreal injection. No drugrelated SAEs were observed.

Neovascular AMD is a significant cause of vision loss. In recent years, VEGF inhibition has become an important strategy in managing the disease. There remains an unmet need in neovascular AMD therapeutics, both in terms of 
enhancing drug activity and in optimizing drug delivery. Aflibercept has a high-binding affinity for VEGF and also binds PIGF with high affinity, characteristics that may distinguish intravitreal aflibercept from other anti-VEGF treatments currently used to treat neovascular AMD. ${ }^{7}$ Currently, all anti-VEGF drugs used to treat neovascular AMD are delivered via the intravitreal injection. Ranibizumab is indicated for monthly injections. However, the intravitreal injection is not without risks. An improvement to the current therapeutic options would be to increase the duration of drug activity, so that intravitreal injections can be administered less frequently. In the current study, a single intravitreal injection of aflibercept provided significant thinning of the thickened retina and lesion at 8 weeks.

The patients enrolled in this primarily safety study had advanced AMD with active neovascularization, intraretinal and/or subretinal fluid, and submacular fibrosis. Despite the advanced stage of disease, CR/LT improved by statistically significant amounts ranging from approximately $80-130 \mu \mathrm{m}$ over 8 weeks post-treatment in the pooled group and trended toward greater improvement in the 4-mg group compared with the 0.15-mg group. Similarly, BCVA demonstrated improvement after treatment in the pooled group, with statistically significant visual gains at 4 and 6 weeks post-treatment; again, a trend was observed toward greater visual gains in the higher dose group. At the 8-week visit, more than twice as many patients in the $0.15-\mathrm{mg}$ group needed redosing compared with patients in the 4-mg group, suggesting that there was a dose-response and that the higher dose of intravitreal aflibercept appeared to have a stronger bioactivity in controlling CNV in neovascular AMD. The fact that fewer patients in the higher dose group met retreatment criteria may also suggest that higher doses of intravitreal aflibercept may provide longer duration of activity in patients with neovascular AMD.

Interestingly, during the first 10-15 days after intravitreal administration of aflibercept, the median decrease in CR/LT was similar between the 2-dose groups, 0.15 and $4 \mathrm{mg}$. However, by week 4 , there was a clear separation between the 2-dose groups, with the higher dose clearly demonstrating superior efficacy over the lower dose. It is possible that initially, the presence of intravitreal aflibercept at both doses was sufficient to bind the VEGF molecules present in the eye, leading to decreased thickness. However, as VEGF continues to be produced over time, a higher concentration of aflibercept may be more capable of continuing to provide sufficient inhibition, resulting in more advantageous outcomes.

The changes in visual acuity over time were less simple to explain. Although beginning at week 4, there was a clear demonstration (Fig. 1B) that the 4-mg dose led to a more significant improvement in visual acuity than the $0.15-\mathrm{mg}$ dose at weeks 6 and 8 , such changes were not obvious during the first 15 days. Within the first 2 weeks, the $0.15-\mathrm{mg}$ dose produced a better visual gain than the 4-mg dose, although both doses produced similar reduction in retinal thickness. The reasons for such observation are not clear, and may be due to the variations seen with a small study population and/or the lower baseline visual acuity in the 0.15-mg group, which may have allowed for initial improvements in visual acuity following intravitreal aflibercept administration.

The difference in bioactivity between the $0.15-\mathrm{mg}$ and the 4 -mg doses is further apparent when considering the 4 randomly selected representative patients. The 2 patients who were treated with the $0.15-\mathrm{mg}$ dose did not show much improvement based on FA and OCT. The CNV remained with active leakage, and OCT showed very little change in the amount of intraretinal and subretinal fluid. On the other hand, the 2 patients who were treated with the higher 4-mg dose clearly demonstrated improvement in the activities of the $\mathrm{CNV}$, with complete resolution of leakage on FA and fluid on OCT. Such results help to provide uncontroversial support for a dose-response effect of intravitreal aflibercept in neovascular AMD. Equally important is the lack of any increase in AEs associated with the higher dose, suggesting that the higher dose of intravitreal aflibercept may have increased bioactivity, but did not carry additional risks of deleterious effects during the 8-week study.

In summary, the current study suggests that intravitreal therapy with $4 \mathrm{mg}$ of aflibercept is well tolerated and has a substantial biological activity that may last up to 8 weeks in patients with CNV secondary to AMD. The study also provides additional data to support a dose-dependent bioactivity of intravitreal aflibercept. It is clear from this shortterm study that the 4-mg dose had an equivalent safety profile with increased bioactivity as compared to the $0.15-\mathrm{mg}$ dose for 8 weeks. Additional studies will help to elucidate optimal dosing and administration frequency with intravitreal aflibercept in patients with neovascular AMD.

\section{Acknowledgments}

This study was sponsored by Regeneron Pharmaceutical, Inc., Tarrytown, NY, and Bayer Healthcare AG.

\section{Author Disclosure Statement}

Financial disclosures are as follows: Dr. Berliner: Equity ownership: Regeneron; current employment by the commercial entity that sponsored the study: Regeneron. Dr. Browning: Current grant support: Regeneron. Dr. Campochiaro: Consulting fees or paid advisory boards: Regeneron. Dr. Cedarbaum: Equity ownership: Regeneron; previous employment by the commercial entity that sponsored the study: Regeneron; patents, royalties: Regeneron. Ms. Chu: Equity ownership: Regeneron; current employment by the commercial entity that sponsored the study: Regeneron. Dr. Do: Current grant support: Regeneron. Dr. Haller: Consulting fees or paid advisory boards: Regeneron. Dr. Hariprasad: Consulting fees: Alcon, Allergan, Bayer, OD-OS, and Optos; member of the Speaker's Bureau for Alcon, Allergan, Regeneron, and Genentech. Dr. Hudson: Consulting fees or paid advisory boards: Regeneron; equity ownership: Regeneron; lecture fees: Regeneron. Dr. Ingerman: Previous employment by the commercial entity that sponsored the study: Regeneron. Dr. Kaiser: Consulting fees or paid advisory boards: Regeneron; lecture fees: Regeneron; current grant support to Cole Eye Inst: Regeneron. Dr. Mieler: No applicable disclosures. Dr. Nguyen: Current grant support: Regeneron. Dr. Shah: No applicable disclosures. Dr. Slakter: Lecture fees: Regeneron; current grant support: Regeneron, Bayer Healthcare. Dr. Sonkin: No applicable disclosures. Dr. Vitti: Equity ownership: Regeneron; current employment by the commercial entity that sponsored the study: Regeneron.

\section{References}

1. Okamoto, N., Tobe, T., Hackett, S.F., et al. Transgenic mice with increased expression of vascular endothelial 
growth factor in the retina: a new model of intraretinal and subretinal neovascularization. Am. J. Pathol. 151:281291, 1997.

2. Kwak, N., Okamoto, N., Wood, J.M., and Campochiaro, P.A. VEGF is major stimulator in model of choroidal neovascularization. Invest. Ophthalmol. Vis. Sci. 41:3158-3164, 2000.

3. Saishin, Y., Saishin, Y., Takahashi, K., et al. VEGF-TRAP(R1R2) suppresses choroidal neovascularization and VEGF-induced breakdown of the blood-retinal barrier. J. Cel.l Physiol. 195:241248, 2003.

4. Krzystolik, M.G., Afshari, M.A., Adamis, A.P., et al. Prevention of experimental choroidal neovascularization with intravitreal anti-vascular endothelial growth factor antibody fragment. Arch Ophthalmol. 120:338-346, 2002.

5. Rosenfeld, P.J., Brown, D.M., Heier, J.S., et al. Ranibizumab for neovascular age-related macular degeneration. N. Engl. J. Med. 355:1419-1431, 2006.

6. Brown, D.M., Kaiser, P.K., Michels, M., et al. Ranibizumab versus verteporfin for neovascular age-related macular degeneration. N. Engl. J. Med. 355:1432-1444, 2006.

7. Holash, J., Davis, S., Papadopoulos, N., et al. VEGF-Trap: a VEGF blocker with potent antitumor effects. Proc. Natl. Acad. Sci. U. S. A. 99:11393-11398, 2002.

8. Nguyen, Q.D., Shah, S.M., Hafiz, G., et al. A phase I trial of an IV-administered vascular endothelial growth factor trap for treatment in patients with choroidal neovascularization due to age-related y. Ophthalmology 113:1522 e1521-1522 e1514, 2006.

9. Stewart, M.W., and Rosenfeld, P.J. Predicted biological activity of intravitreal VEGF Trap. Br. J. Ophthalmol. 92:667668, 2008.

10. Nguyen, Q.D., Shah, S.M., Browning, D.J., et al. A phase I study of intravitreal vascular endothelial growth factor trapeye in patients with neovascular age-related macular degeneration. Ophthalmology 116:2141-2148 e2141, 2009.

11. Laser photocoagulation of subfoveal neovascular lesions in age-related macular degeneration. Results of a randomized clinical trial. Macular Photocoagulation Study Group. Arch Ophthalmol. 109:1220-1231, 1991.

Received: December 27, 2011

Accepted: June 4, 2012

Address correspondence to: Prof. Quan Dong Nguyen The Wilmer Eye Institute The Johns Hopkins University School of Medicine 600 North Wolfe Street Maumenee 745

Baltimore, MD 21287-9277

E-mail: qnguyen4@jhmi.edu 